\title{
miR-133a suppresses cell proliferation, migration and invasion in human lung cancer by targeting MMP-14
}

\author{
MENG XU ${ }^{1 *}$ and YU-ZHOU WANG ${ }^{1,2^{*}}$ \\ ${ }^{1}$ Department of Oncology, The First Affiliated Hospital, Jinan University, Guangzhou, Guangdong 510630; \\ ${ }^{2}$ Cancer Center of Affiliated Hospital of Guangdong Medical College, Zhanjiang, Guangdong 524002, P.R. China
}

Received February 16, 2013; Accepted May 17, 2013

DOI: $10.3892 /$ or.2013.2548

\begin{abstract}
Lung cancer is the leading cause of cancer-related mortality worldwide. Over half of lung cancer cases are diagnosed after metastasis, for which the median survival time is approximately 8 months. microRNAs (miRNAs), which are a class of single-stranded endogenous non-coding RNAs, are likely to be involved in most biological processes. miR-133 plays roles in cardiac development and disease, and recent studies showed that miR-133 is downregulated in various human malignancies, such as bladder and lung cancer. However, its detailed role in the processes of cancer remains to be determined. In the present study, we found that in the lung cancer cell lines A549 and NCI-H1299 overexpression of miR-133a suppressed cell proliferation, migration and invasion. The miR-133a-induced suppression of cell migration and invasion can be reversed by miR-133a-specific inhibitor. According to the mRNA sequence, matrix metalloproteinase (MMP)-14, which is an important regulator of metastasis, is a predicted target of miR-133a. This was confirmed by dual luciferase reporter assay. Moreover, miR-133a overexpression decreases the mRNA and protein levels of MMP-14. Collectively, these results suggest that miR-133a may inhibit lung cancer metastasis by targeting MMP-14 and may be used as an anti-metastatic therapy in lung cancer patients.
\end{abstract}

Correspondence to: DrMeng Xu, Department of Oncology, The First Affiliated Hospital, Jinan University, West no. 613 Huangpu Road, Guangzhou, Guangdong 510630, P.R. China

E-mail: xumengjinan1314@163.com

Dr Yu-Zhou Wang, Cancer Center of Affiliated Hospital of Guangdong Medical College, South 57 People's Road, Xiashan, Zhanjiang, Guangdong 524002, P.R. China

E-mail:072101023@fudan.edu.cn

${ }^{*}$ Contributed equally

Abbreviations: MMP, matrix metalloproteinase; MT-MMP, membrane-type MMP; miRNA, microRNA; PVDF, polyvinylidene difluoride membrane

Key words: microRNA-133a, matrix metalloproteinase-14, cell proliferation, invasion

\section{Introduction}

Metastasis, which is the spread of cancer to distant locations in the body, is a complex process. Approximately $90 \%$ of deaths associated with cancer are due to the metastasis of the original tumor cells to sites distant from the primary tumor. Metastasis occurs through a series of sequential steps including invasion of adjacent tissues, intravasation, transport of cancer cells through the circulatory system, arrest at a secondary site, extravasation and growth in a secondary organ (1). For most cancer cell types, the ability to metastasize leads to clinically incurable disease (2). However, the mechanisms underlying a cell's ability to extravasate from the primary tumor, circulate and invade new tissue remain to be established (3). The organized breakdown of the extracellular matrix (ECM) by matrix metalloproteinases (MMPs) is involved in the complex cascade of events, such as invasion and extravasation. The human MMP family is divided into secreted and membrane-type MMPs (MT-MMPs). MT-MMPs are membrane-tethered proteolytic enzymes and constitute the largest family of MMPs identified thus far (4). The type I transmembrane structural class of MT-MMPs contains MMP-14 (MT1-MMP), MMP-15 (MT2-MMP), MMP-16 (MT3-MMP) and MMP-24 (MT5-MMP). Overexpression of MT-MMPs has been observed in several types of human cancer (5). The functions of MT-MMPs are known to include roles in activation of other MMP family members, pericellular proteolysis, modulation of cellular signaling and cellular migration, the angiogenic response and regulation of cell proliferation, apoptosis and tumor metastasis (6-8). Among these members, MMP-14 is overexpressed in pancreatic ductal adenocarcinoma (PDAC) compared with normal pancreatic tissue, which is induced by TGF- $\beta 1$ (9). The overexpression of MMP-14 is also observed in non-small cell lung carcinoma (NSCLC) (10), breast cancer (11), malignant mesothelioma (12), and supraglottic cancer (13). The activity of these proteases is tightly regulated by specific inhibitors, known as tissue inhibitors of MMPs (TIMPs) (14). A selective MMP-14 inhibitor reduces cancer cell motility and tumor growth (15). Therefore, to uncover the regulation mechanisms of MMP-14, particularly in cancer metastasis, is of great importance for understanding cancer biology and improving treatment.

microRNAs (miRNAs) are 20-23-nucleotide long singlestranded RNAs that are encoded by eukaryotic nuclear DNA 
and function in the post-transcriptional regulation of gene expression via base-pairing with complementary sequences within 3'-UTR of target mRNAs (16). Mature miRNAs, Argonaute (Ago) and several other associated proteins form the RNA-induced silencing complex (RISC) mediating gene silencing at post-transcriptional and translational levels (17). miRNAs have been shown to be involved in a wide variety of biological processes such as apoptosis, development, aging and cancer. Previous studies suggested that miRNAs contribute to the initiation and development of various types of cancer (18). There is also accumulating evidence to suggest that miRNAs are involved in tumor metastasis. miR-200 family and miR-205 can inhibit epithelial-mesenchymal transition (EMT), through which epithelial cancer cells invade and metastasize, by targeting ZEB1, ZEB1 and SIP1 (19-21). In human breast cancer, overexpression of miR-21 is significantly correlated with lymph node metastasis, advanced clinical stage and shortened survival time (22). miR-10b is also reported to initiate tumor invasion and metastasis in breast cancer $(23,24)$. In prostate cancer progenitor cells, miR-34a acts as a potent inhibitor of metastasis by directly suppressing CD44 (25). miR-9, an MYC/MYCN-activated miRNA, is reported to enhance cell motility and invasiveness by targeting CDH1 (26). Therefore, it is reasonable to assume that more miRNAs would be discovered to govern metastasis. miR-133a has previously been reported to play roles in diabetic hearts (27), myogenic differentiation (28) and osteoblast lineage commitment program (29). Recent findings showed that miR-1 and miR-133a are frequently downregulated in various types of cancer (30). In the present study, we proved that MPP-14 is a target of miR-133a by dual luciferase reporter assay, and overexpression of miR-133a decreases the mRNA and protein levels of MMP-14 in lung cancer cell lines. In conclusion, the miR-133a-induced suppression of cell proliferation, migration and invasion addresses the anti-metastatic role of miR-133a in lung cancer.

\section{Materials and methods}

Cell culture and transfection. NSCLC cell lines A549, NCI-H1299 and human HEK-293T cell lines were purchased from the American Type Culture Collection (ATCC, Rockville, MD, USA). The cell lines were cultured in Dulbecco's modified Eagle's medium (DMEM; Invitrogen, Carlsbad, CA, USA) supplemented with $10 \%$ fetal bovine serum (FBS) (S/N:16000-044; Gibco) and antibiotics (100 U/ml penicillin and streptomycin; Invitrogen) at $37^{\circ} \mathrm{C}$ in a humidified $5 \%$ $\mathrm{CO}_{2}$ atmosphere. Cell transfection was performed using Lipofectamine $^{\mathrm{TM}} 2000$ (Invitrogen). Cells were harvested at $48 \mathrm{~h}$ post-transfection for protein analysis or luciferase activity assay. The related sequences are shown in Table I.

Plasmid construction. The full length 3'UTR of human MMP-14 was PCR-amplified from human genomic DNA and cloned into psiCHECK ${ }^{\mathrm{TM}}-2$ dual luciferase reporter plasmid (Promega, Madison, WI, USA) immediately downstream of the stop codon of the Renilla luciferase gene with Xhol/NotI to generate psiCHECK ${ }^{\mathrm{TM}}$-2-MMP-14-3'UTR-wt. The mutant human MMP-14 3'UTR reporter, designated as psiCHECK ${ }^{\mathrm{TM}}$-2-MMP-14-3'UTR-mut, was created within
Table I. Primers used for reverse transcription and real-time polymerase chain reaction.

\begin{tabular}{ll}
\hline Primer & \multicolumn{1}{c}{ Sequence } \\
\hline $\begin{array}{l}\text { miR-133a-stem-loop } \\
\text { RT primer }\end{array}$ & GTA TTC GCA CTG GAT ACG ACT CAG \\
& CTC-3' \\
RNU6-RT & 5'-AAA ATA TGG AAC GCT-3' \\
miR-133a mimics & 5'-TTT GGT CCC CTT CAA CCA GCT G-3' \\
Mimics control & 5'-AGT GTG AGT TCT ACC ATT GCC \\
& AAA-3' \\
miR-133a inhibitor & 5'-CAG CTG GTT GAA GGG GAC CAAA-3' \\
& (2'Ome) \\
miR-133a-F & 5'-GTG CAT TTG GTC CCC TTC A-3' \\
miR-133a-R & 5'-CGG GCT GTC AGT TTG TCA-3' \\
RNU6-F & 5'-CTC GCT TCG GCA GCA CA-3' \\
RNU6-R & 5'-AAC GCT TCA CGA ATT TGC GT-3' \\
GAPDH-F & 5'-AGG GCA TCT TGG GCT ACA C-3' \\
GAPDH-R & 5'-TGG TCC AGG GTT TCT TAC TCC-3' \\
MMP-14-F & 5'-GCA GAA GTT TTA CGG CTT GCA-3' \\
MMP-14-R & 5'-TCG AAC ATT GGC CTT GAT CTC-3' \\
\hline
\end{tabular}

F, forward; R, reverse; GAPDH, glyceraldehyde-3-phosphate dehydrogenase; MMP, matrix metalloproteinase.

the predicted hsa-miR-133a seed sequence binding site

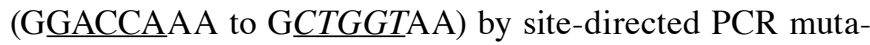
tion. The construction was confirmed by DNA sequencing.

Luciferase reporter assay. For luciferase reporter assay, HEK-293T cells $\left(4.0 \times 10^{3}\right.$ cells/well) were plated in a 96-well plate (Corning, USA) $24 \mathrm{~h}$ before transfection. Cells were co-transfected with $60 \mathrm{ng}$ of either the psiCHECK ${ }^{\mathrm{TM}}-2$ MMP-14-3'UTR-wt, psiCHECK ${ }^{\mathrm{TM}}$-2-MMP-14-3'UTR-mut or psiCHECK ${ }^{\mathrm{TM}}-2$ vector and $20 \mathrm{nM}$ (final concentration) of either miRNA mimics negative control or miR-133a mimics or miR-133a inhibitor, which is antisense oligonucleotides with 2'O-methyl modification (RiboBio, Guangzhou, China). Forty-eight hours after transfection, the cells were washed with PBS twice and lysed in $100 \mu \mathrm{l}$ Passive Lysis Buffer (Promega) and the luciferase activities were measured from $20 \mu$ l lysate using the Dual-Luciferase Reporter Assay kit (Promega) following the manufacturer's instructions on a luminometer (Lumat LB 9507; Berthold, Germany). The data were obtained by averaging the results from six independent repeats. Transfection efficiency was normalized to thymidine kinase-driven Renilla luciferase activity.

$R N A$ isolation and real-time PCR. Total RNA was isolated from cultured cells using TRIzol Reagent (Invitrogen). cDNA was synthesized by reverse transcription using oligo (dT) as the primer and proceeded to real-time PCR with gene-specific primers in the presence of 2X SYBR-Green Master Mix (DBI Bioscience, Shanghai, China). The relative abundance of mRNA was calculated by normalization to glyceraldehyde-3-phosphate dehydrogenase (GAPDH) mRNA. Specific stem-loop 
A

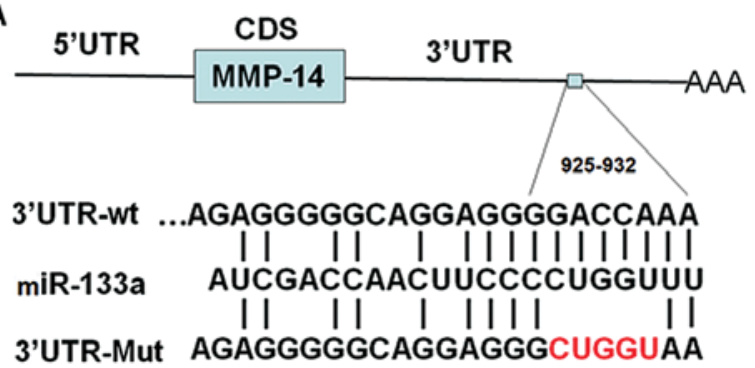

B

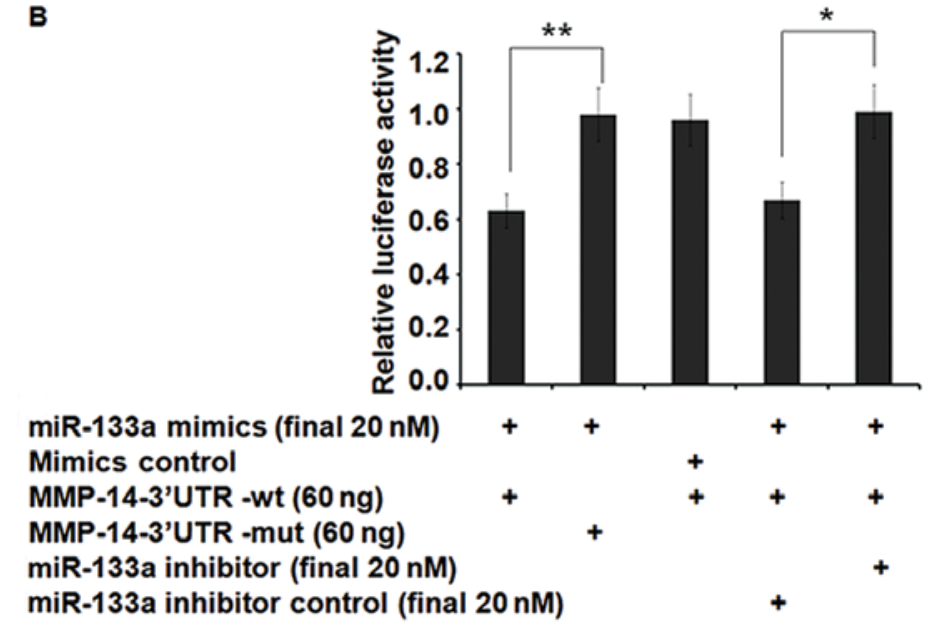

Figure 1. Matrix metalloproteinase (MMP)-14 is a target of miR-133a. (A) The highly conserved mature miR-133a sequence in mammals and the potential seed matching between miR-133a and MMP-14 3'UTR sequence are shown. The mutated bases in MMP-14-3'UTR-mut are indicated in red. (B) The transfection was performed as indicated in $293 \mathrm{~T}$ cells, followed by dual luciferase activity assay $48 \mathrm{~h}$ later. Data of three independent experiments are presented as mean $\pm \mathrm{SE} .{ }^{* *} \mathrm{P}<0.01,{ }^{*} \mathrm{P}<0.05$.

RT primers were used for reverse transcription reaction of miR-133a. Normalization of miR-133a was performed by using RNU6 primers. All reactions were carried out in triplicate and all experiments were performed three independent times.

Proliferation, migration and Matrigel invasion assay. For the proliferation assay, $6 \mathrm{~h}$ after transfection, 3,000 cells were seeded in triplicate in 6-well plates. Cell Counting Kit-8 (CCK-8) reagent (C0038; Beyotime, China) was added at 12, $24,48,72 \mathrm{~h}$ after seeding and incubated at $37^{\circ} \mathrm{C}$ for half to $4 \mathrm{~h}$ according to the color change. The data of optical density (OD) value at $450 \mathrm{~nm}$ were read by a microplate reader (Synergy 2; BioTek, USA). Migration and invasion assays were performed according to the manufacturer's instructions. Cells $\left(3 \times 10^{4}\right)$ in $0.5 \mathrm{ml}$ of serum-free medium were seeded onto the top of each chamber containing BD BioCoat cell culture inserts (354578) or Matrigel Invasion Chamber (354480; both from BD Biosciences), and $0.75 \mathrm{ml}$ of complete growth medium containing $10 \%$ FBS was added to each well in the lower chamber. Following incubation for $48 \mathrm{~h}$ at $37^{\circ} \mathrm{C}$, non-invasive cells were removed from the upper chamber, the cells attached to the lower chamber were fixed with methanol, stained with $0.1 \%$ crystal violet and then counted under a light microscope.

Western blot analysis. Fifty micrograms of total proteins were loaded onto $10 \%$ sodium dodecyl sulfate-polyacrylamide gel electrophoresis and transferred onto polyvinylidene difluoride membranes (PVDF; Millipore, Billerico, MA, USA). After blocking with 3\% bovine serum albumin, the blots were incubated with antibodies against MMP-14 (Bioworld, Atlanta, GA, USA), GAPDH (Chemicon International, Temecula, CA, USA). Following incubation with red flurescence conjugated secondary antibody, protein bands were scanned using Odyssey bands scanner (S/N ODY-2792 model: 9120) The intensity of the bands was analyzed using Bandscan Software.

Statistical analysis. Analysis of variance and Student's t-test were used to compare means of two or more different treatment groups. $\mathrm{P}<0.05$ was considered to indicate a statistically significant difference, unless otherwise stated. Data are expressed as mean \pm SE.

\section{Results}

$M M P-14$ is a functional target of miR-133a. To identify potential targets of miR-133a both for experimental validation and functional studies in lung cancer, we performed in silico analysis of a range of miRNA target prediction databases. The target prediction of miR-133a was performed by using the following databases: TargetScan (http://www.targetscan.org), MicroCosm (http://www.ebi.ac.uk/), miRanda (http://www. microrna.org/microrna/getGeneForm.do) and miRGen (www. diana.pcbi.upenn.edu/cgi-bin/miRGen/v3/Targets. cgi). All databases analyzed presented MMP-14 as a converging target of miR-133a, which plays an essential role in the degradation of basement membranes and the expression of MMP-14 is correlated with metastasis. To validate MMP-14 as a de novo target of miR-133a, we constructed a luciferase reporter vector containing the entire wild-type 3'UTR of MMP-14 (psiCHECK ${ }^{\mathrm{TM}}-2$-MMP-14-3'UTR-wt) or the mutant 3'UTR (psiCHECK ${ }^{\mathrm{TM}}$-2-MMP-14-3'UTR-mut) with a 5-nucleotide mutation previously mentioned (Fig. 1A). HEK-293T cells, which exhibit low levels of miR-133a expression, were used for transient transfections with psiCHECK $^{\mathrm{TM}}-2$-MMP14-3'UTR-wt or psiCHECK ${ }^{\mathrm{TM}}$ 2-MMP-14-3'UTR-mut. Co-transfection with miR-133a (a synthetic miR-133a mimic) resulted in a significant decrease (to 63\%) in luciferase gene expression from the reporter vector containing the wild-type 3'UTR of MMP-14 when compared with a scrambled control (Fig. 1B), demonstrating direct targeting by miR-133a $(\mathrm{P}<0.01)$. Consistent with the data, no decrease in luciferase activity was observed when miR-133a was co-transfected with the mutant 3'UTR of MMP-14 reporter. Furthermore, miR-133a specific inhibitor, which is the antisense oligonucleotides of miR-133a can almost restore the inhibition effect $(\mathrm{P}<0.05)$ (Fig. 1B). These results indicate that MMP-14 is a direct target 
A

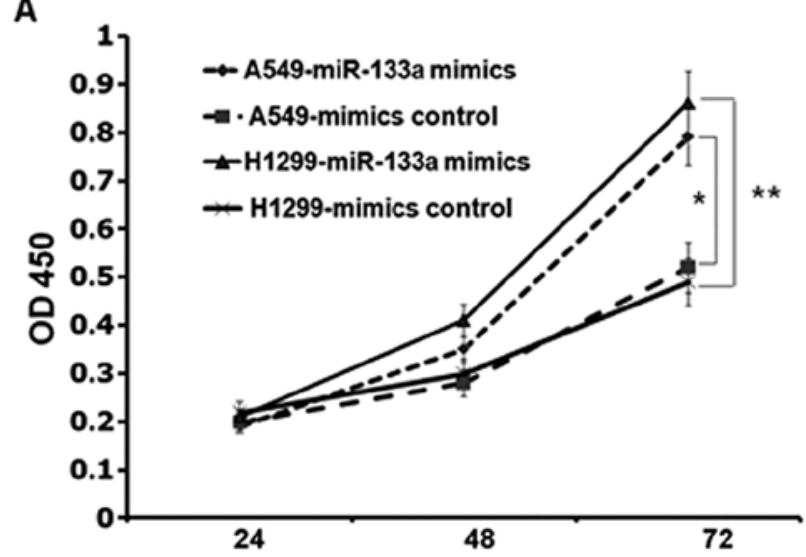

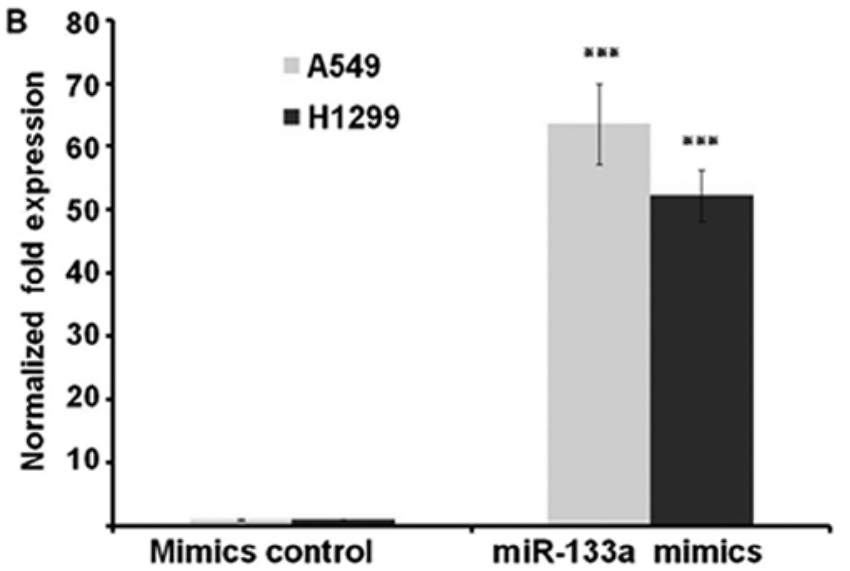

Figure 2. miR-133a suppresses cell proliferation in lung cancer cells. (A) Growth curves of A549 and NCI-H1299 cells after transfection as indicated were determined by Cell Counting Kit-8. (B) Mature miR-133a was determined using quantitative PCR with specific stem-loop reverse transcription primer after transfection.

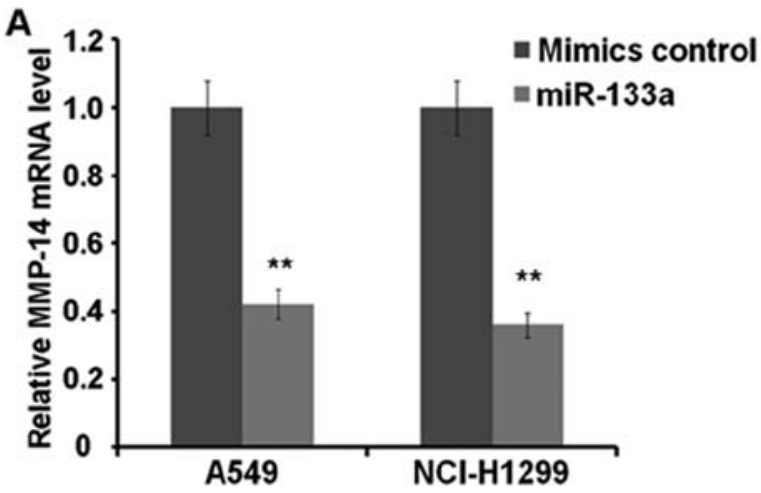

B

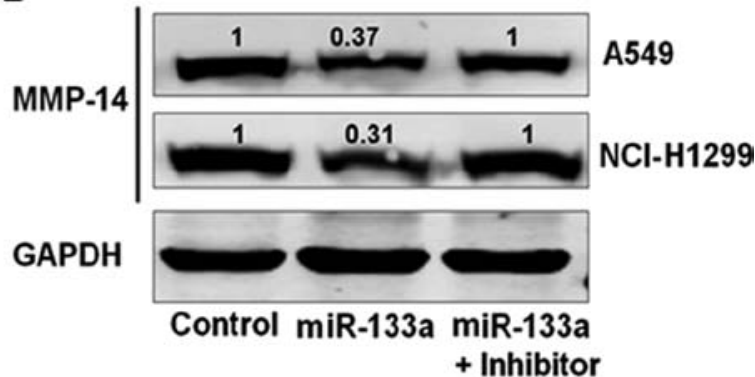

Figure 3. miR-133a decreases the mRNA and protein levels of matrix metalloproteinase (MMP)-14 (A) Total RNA was extracted from A549 and NCI-H1299 cells after transfection as indicated, and quantitative PCR was performed to determine MMP-14 expression. (B) A549 and NCI-H1299 cells were transfected as indicated. Endogenous MMP-14 and PCNA protein levels were determined by western blotting along with the glyceraldehyde-3-phosphate dehydrogenase (GAPDH) control. Relative protein levels were normalized by GAPDH and quantified by the ratio compared with controls.

of miR-133a and is responsible for miR-133a targeting in the 3'UTR.

miR-133a suppresses cell proliferation in lung cancer A549 and NCI-H1299 cell lines. To characterize the functional impact of miR-133a on lung cancer cell behavior such as growth rate, we transfected A549 and NCI-H1299 cells with either miR-133a mimics or mimics control and transferred the cells into 96 -well plates at $3.0 \times 10^{3}$ cells/well post transfection. We harvested and detected the OD450 value of each well by CCK- 8 kit at indicated times after seeding. These results showed that the growth rates of the two lung cancer cell lines were suppressed in miR-133a mimics transfected groups compared to control groups at $72 \mathrm{~h}(\mathrm{P}<0.01$ and $\mathrm{P}<0.05$ at $\mathrm{A} 549$ and NCI-H1299 cell lines, respectively) (Fig. 2A). To confirm the transfection, we assessed the expressions of miR-133a at $72 \mathrm{~h}$ post transfection. As shown in Fig. 2B, miR-133a was upregulated to 63.6- and 52.3-fold at A549 and NCI-H1299 cells, respectively (both $\mathrm{P}<0.001$ ) (Fig. 2B).

Overexpression of $m i R-133$ a decreases the $m R N A$ and protein levels of MMP-14. We next assessed the effect of miR-133a overexpression on MMP-14. Transfection of miR-133a into A549 and NCI-H1299 cells resulted in a significant decrease of MMP-14 mRNA levels compared with control-transfected cells. As measured by qRT-PCR, the MMP-14 mRNA levels were decreased to 41 and $38 \%$ in A549 and NCI-H1299, respectively (both $\mathrm{P}<0.01$ ) (Fig. 3A). Subsequent western blot analysis of MMP-14 showed that miR-133a overexpression caused a reduction in MMP-14 protein levels, which can be reversed by miR-133a specific inhibitor (Fig. 3B). Measured by Bandscan Software according to grayscale of each band, MPP-14 protein is downregulated to 37 and 31\% in A549 and NCI-H1299 cells, respectively. These results indicated that miR-133a can inhibit lung cancer cell proliferation, which may be mediated by downregulation of direct target MMP-14 at mRNA and protein levels.

miR-133a inhibits lung cancer cell migration and invasion through targeting MMP-14. MMP-14 is an MT1-MMP as a primary regulator of interstitial collagenolysis and there is evidence to suggest that MMP-14 is enhanced in metastatic PDAC lesions compared with the primary tumors and myogenic tumors $(31,32)$. The downregulation of MMP-14 by miR-133a 
A

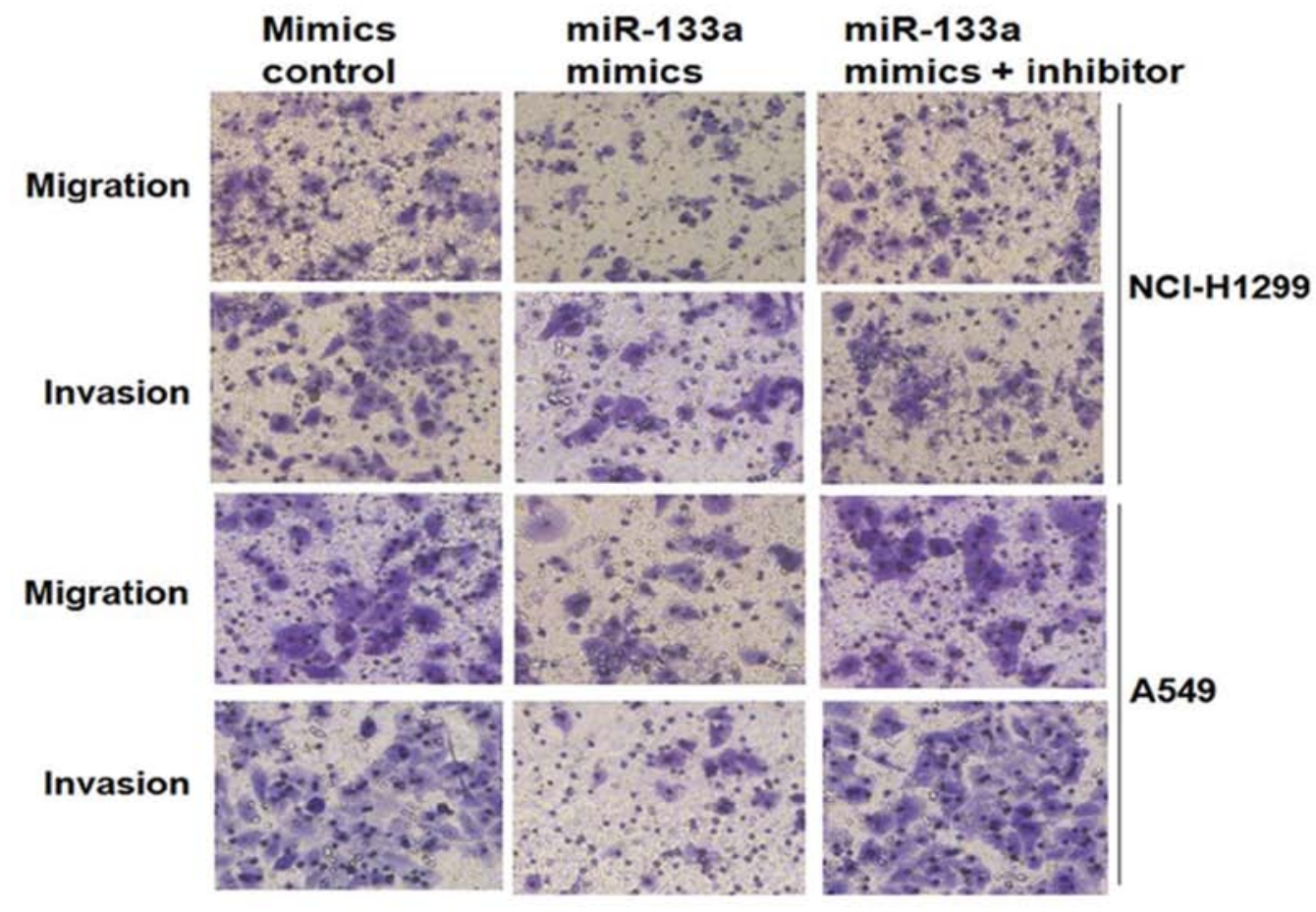

B

E Control $=$ Mimics $=$ Mimics + Inhibitor

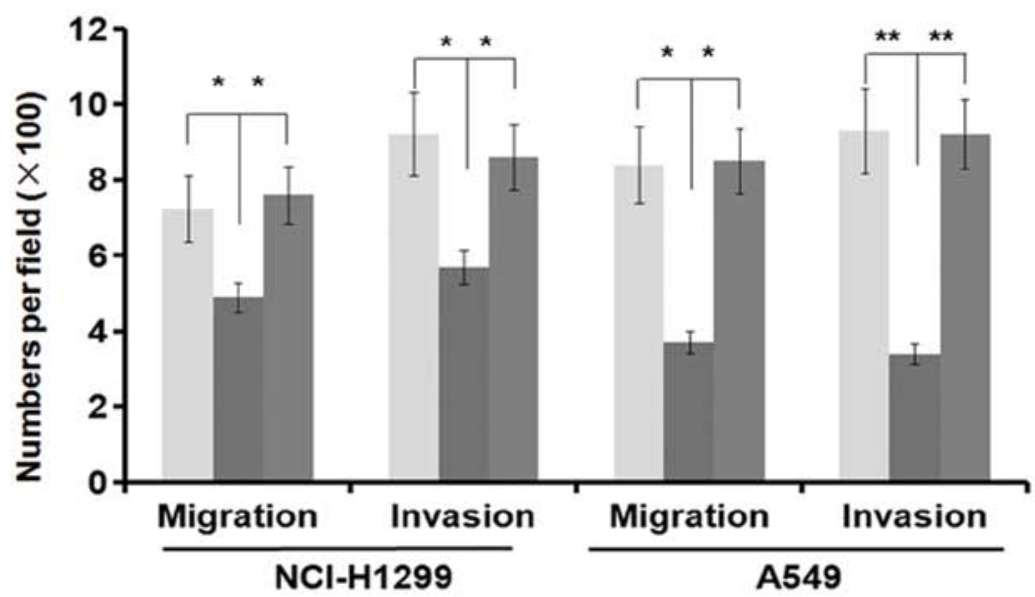

Figure 4. miR-133a inhibits cell migration and invasion in lung cancer cell lines and the miR-133a specific inhibitor reverses the effect. (A) A549 and NCI-H1299 cells were subjected to transwell migration and Matrigel invasion assays $6 \mathrm{~h}$ after transfection as indicated. The crystal violet dye staining images of the lower chambers are shown. (B) The data are shown as mean $\pm \mathrm{SE}$ of the number of cells that migrated or invaded in $\mathrm{n}=6$ separate experiments. ${ }^{* *} \mathrm{P}<0.01$ and ${ }^{* * *} \mathrm{P}<0.001$ as compared with controls. Magnification, $\mathrm{x} 100$.

overexpression prompted us to examine whether miR-133a could modulate migration and invasion in lung cancer cell lines. We performed the transwell migration and Matrigel invasion assays in A549 and NCI-H1299 cells transfected with either miR-133a mimics or control mimics. As shown in Fig. 3A, miR-133a mimics suppressed cell migration and invasion in both A549 and NCI-H1299 cells (migration and invasion, $\mathrm{P}<0.05$ in the NCI-H1299 cell line and $\mathrm{P}<0.01$ in the A549 cell line) (Fig. 4A). The specific inhibitor of miR-133a almost restored the inhibition effect in both cell lines (Fig. 4A and B). Moreover, the MMP-14 protein levels were also restored (Fig. 3B). Collectively, these results clearly indicate that miR-133a suppresses cell migration and invasion in the A549 and NCI-H1299 cells lines by reducing MMP-14 expression.

\section{Discussion}

miRNAs play important roles in a broad range of biological processes including development, cellular differentiation, proliferation and apoptosis (18). Increasing evidence shows that several miRNAs including the miR-200 family, miR-10b and miR-205 are involved in the lung cancer metastasis process (19-21). In the present study, we identified that miR-133a is another miRNA which can inhibit cell proliferation, migration and invasion in lung cancer cell lines by targeting MMP-14. Several lines of evidence support this idea. First, downregulation of MMP-14 by miR-133a was confirmed in A549 and NCI-H1299 cell lines at the protein and mRNA levels. Second, miR-133a repressed luciferase 
reporter gene containing the MMP-14 3'UTR through its binding site and mutation of the putative binding site on MMP-14 3'UTR almost abolished the suppression effect. Third, overexpression of miR-133a in A549 and NCI-H1299 cells suppressed cell proliferation, migration and invasion through reducing MMP-14 expression. Moreover, the specific antisense miR-133a inhibitor can almost restore the inhibition effect as well.

A number of recent studies analyzed the genetic expression of MMP-14 in several types of human cancer, including tumors of the breast, colon, head, neck and oral cavity $(6,8)$, indicating the importance of MMP-14 in cancer progression. MMP-14 is an essential molecule whose function is known to include roles in the activation of other MMP family members, pericellular proteolysis, modulation of cellular signaling and cell migration (5). It is not surprising that the expression of MMP-14 is tightly regulated at multiple levels. For example, TGF- $\beta 1$ was reported to promote migration in an MMP-14 dependent manner in pancreatic ductal epithelial (HPDE) cells (9). In the present study, we confirmed that MMP-14 is indeed a functional target of miR-133a and found that the expression of MMP-14 is negatively regulated by miR-133a. It should be noted that mature miR-133a sequence (5'-UUUGGU CCCCUUCAACCAGCUA-3') is highly conserved among mammalian species. The potential miR-133a binding site (5'-UUUGGUC-3') is also presented in the known MMP-14 3'UTR of rat, dog, horse and cattle, suggesting that miR-133a may regulate MMP-14 expression of these species in a similar manner. Collectively, it is reasonable to hypothesize that posttranscriptional regulation of MMP-14 by miRNAs may be a major determinant of MMP-14 expression in lung cancer growth and metastasis.

The functions of mammalian miR-133a and its targets have been reported in several publications. Ectopic expression of miR-133a significantly inhibited the invasion capacity of various human cancer cell lines $(27,28)$. It is reported that miR-133a targets FSCN1 in MCF-7 and MDA-MB-231 breast cancer cell lines. In breast cancer, miR-133a expression was found reduced by a microarray-based analysis (27), which indicated the tumor suppressive function of miR-133a. As is known, miRNAs can target hundreds of targets (16), miRNAs may play different roles in different cell lines or under different signals. We cannot exclude the possibility that a single miRNA can exert multiple functions by targeting multiple targets. In the present study, ectopic expression of miR-133a was more than 50-fold compared with endogenous expression, but MMP-14 is only downregulated to approximately $30 \%$, indicating that the endogenous expression of miR-133a is extremely low and that MMP-14 may not be the only target in this condition. The specific inhibitor of miR-133a can almost restore the suppression of cell migration and invasion. These results suggest the function of miR-133a in lung cancer is prominently inhibition of tumor metastasis rather than tumor growth.

In summary, in the present study we described for the first time that miR-133a plays an anti-metastatic role in lung cancer and miR-133a may be a suitable tumor marker for lung cancer metastasis. We also proved that MMP-14 is a new functional target of miR-133a. In further studies, we will uncover the complex functions of miR-133a in modulating lung cancer progression.

\section{Acknowledgements}

The present study was supported by the National Natural Science Foundation of China (81273814).

\section{References}

1. Mehlen P and Puisieux A: Metastasis: a question of life or death. Nat Rev Cancer 6: 449-458, 2006.

2. Steeg PS: Metastasis suppressors alter the signal transduction of cancer cells. Nat Rev Cancer 3: 55-63, 2003.

3. Segura MF, Hanniford D, Menendez S, et al: Aberrant miR-182 expression promotes melanoma metastasis by repressing FOXO3 and microphthalmia-associated transcription factor. Proc Natl Acad Sci USA 106: 1814-1819, 2009.

4. Egeblad $M$ and Werb Z: New functions for the matrix metalloproteinases in cancer progression. Nat Rev Cancer 2: 161-174, 2002.

5. Yana I and Seiki M: MT-MMPs play pivotal roles in cancer dissemination. Clin Exp Metastasis 19: 209-215, 2002

6. Genís L, Gálvez BG, Gonzalo P and Arroyo AG: MT1-MMP: universal or particular player in angiogenesis? Cancer Metastasis Rev 25: 77-86, 2006.

7. Plaisier M, Kapiteijn K, Koolwijk P, et al: Involvement of membrane-type matrix metalloproteinases (MT-MMPs) in capillary tube formation by human endometrial microvascular endothelial cells: role of MT3-MMP. J Clin Endocrinol Metab 89: 5828-5836, 2004.

8. Sounni NE and Noel A: Membrane type-matrix metalloproteinases and tumor progression. Biochimie 87: 329-342, 2005.

9. Ottaviano AJ, Sun L, Ananthanarayanan V and Munshi HG: Extracellular matrix-mediated membrane-type 1 matrix metalloproteinase expression in pancreatic ductal cells is regulated by transforming growth factor- $\beta 1$. Cancer Res 66: 7032-7040, 2006.

10. Atkinson JM, Pennington CJ, Martin SW, et al: Membrane type matrix metalloproteinases (MMPs) show differential expression in non-small cell lung cancer (NSCLC) compared to normal lung: correlation of MMP-14 mRNA expression and proteolytic activity. Eur J Cancer 43: 1764-1771, 2007.

11. Laudański P, Swiatecka J, Kozłowski L, et al: Increased serum level of membrane type 1-matrix metalloproteinase (MT1-MMP/ MMP-14) in patients with breast cancer. Folia Histochem Cytobiol 48: 101-103, 2010.

12. Crispi S, Calogero RA, Santini M, et al: Global gene expression profiling of human pleural mesotheliomas: identification of matrix metalloproteinase 14 (MMP-14) as potential tumour target. PLoS One 4: e7016, 2009.

13. Zhang H, Liu M, Sun Y and Lu J: MMP-14 can serve as a prognostic marker in patients with supraglottic cancer. Eur Arch Otorhinolaryngol 266: 1427-1434, 2009.

14. Visse R and Nagase H: Matrix metalloproteinases and tissue inhibitors of metalloproteinases: structure, function, and biochemistry. Circ Res 92: 827-839, 2003.

15. Suojanen J, Salo T, Koivunen E, et al: A novel and selective membrane type-1 matrix metalloproteinase (MT1-MMP) inhibitor reduces cancer cell motility and tumor growth. Cancer Biol Ther 8: 2362-2370, 2009.

16. Winter J, Jung S, Keller S, et al: Many roads to maturity: microRNA biogenesis pathways and their regulation. Nat Cell Biol 11: 228-234, 2009.

17. Eulalio A, Huntzinger E and Izaurralde E: Getting to the root of miRNA-mediated gene silencing. Cell 132: 9-14, 2008.

18. Calin GA and Croce CM: MicroRNA signatures in human cancers. Nat Rev Cancer 6: 857-866, 2006.

19. Gregory PA, Bert AG, Paterson EL, et al: The miR-200 family and miR-205 regulate epithelial to mesenchymal transition by targeting ZEB1 and SIP1. Nat Cell Biol 10: 593-601, 2008.

20. Renthal NE, Chen CC, Williams KC, et al: miR-200 family and targets, ZEB1 and ZEB2, modulate uterine quiescence and contractility during pregnancy and labor. Proc Natl Acad Sci USA 107: 20828-20833, 2010.

21. Burk U, Schubert J, Wellner U, et al: A reciprocal repression between ZEB1 and members of the miR-200 family promotes EMT and invasion in cancer cells. EMBO Rep 9: 582-589, 2008.

22. Yan LX, Huang XF, Shao Q, et al: MicroRNA miR-21 overexpression in human breast cancer is associated with advanced clinical stage, lymph node metastasis and patient poor prognosis. RNA 14: 2348-2360, 2008. 
23. Ma L, Teruya-Feldstein $\mathrm{J}$ and Weinberg RA: Tumour invasion and metastasis initiated by microRNA-10b in breast cancer. Nature 449: 682-688, 2007.

24. Gee HE, Camps C, Buffa FM, et al: MicroRNA-10b and breast cancer metastasis. Nature 455: E8-E9, 2008.

25. Liu C, Kelnar K, Liu B, et al: The microRNA miR-34a inhibits prostate cancer stem cells and metastasis by directly repressing CD44. Nat Med 17: 211-215, 2011.

26. Ma L, Young J,Prabhala H, et al: $\mathrm{miR}-9$, a MYC/MYCN-activated microRNA, regulates E-cadherin and cancer metastasis. Nat Cell Biol 12: 247-256, 2010.

27. Xiao J, Luo X, Lin H, et al: MicroRNA miR-133 represses HERG $\mathrm{K}^{+}$channel expression contributing to QT prolongation in diabetic hearts. J Biol Chem. 282: 12363-12367, 2007.

28. Kato Y, Miyaki S, Yokoyama S, et al: Real-time functional imaging for monitoring miR-133 during myogenic differentiation. Int J Biochem Cell Biol 41: 2225-2231, 2009.
29. Li Z, Hassan MQ, Volinia S, et al: A microRNA signature for a BMP2-induced osteoblast lineage commitment program. Proc Natl Acad Sci USA 105: 13906-13911, 2008.

30. Yoshino H, Chiyomaru T, Enokida H, et al: The tumour-suppressive function of miR-1 and miR-133a targeting TAGLN2 in bladder cancer. Br J Cancer 104: 808-818, 2011.

31. Shields MA, Dangi-Garimella S, Krantz SB, et al: Pancreatic cancer cells respond to type I collagen by inducing snail expression to promote membrane type 1 matrix metalloproteinase-dependent collagen invasion. J Biol Chem 286: 10495-10504, 2011

32. Gong YL, Xu GM, Huang WD and Chen LB: Expression of matrix metalloproteinases and the tissue inhibitors of metalloproteinases and their local invasiveness and metastasis in Chinese human pancreatic cancer. J Surg Oncol 73: 95-99, 2000. 